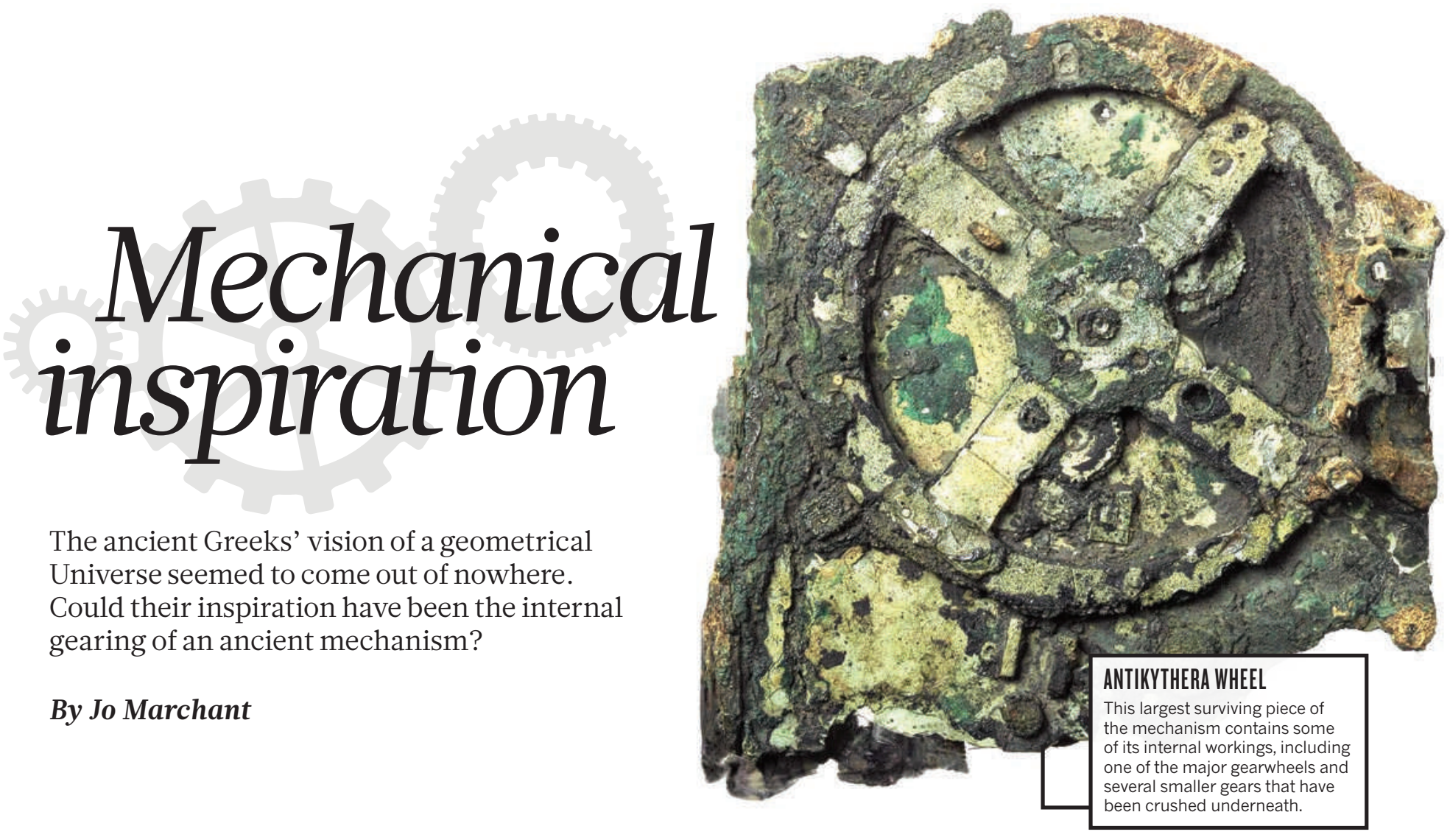

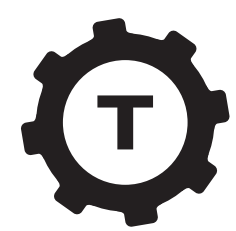

wo thousand years ago, a Greek mechanic set out to build a machine that would model the workings of the known Universe. The result was a complex clockwork mechanism that displayed the motions of the Sun, Moon and planets on precisely marked dials. By turning a handle, the creator could watch his tiny celestial bodies trace their undulating paths through the sky.

The mechanic's name is now lost. But his machine, dubbed the Antikythera mechanism, is by far the most technologically sophisticated artefact that survives from antiquity. Since a reconstruction of the device hit the headlines in 2006, it has revolutionized ideas about the technology of the ancient world, and has captured the public imagination as the apparent pinnacle of Greek scientific achievement.

Now, however, scientists delving into the astronomical theories encoded in this quintessentially Greek device have concluded that they are not Greek at all, but Babylonian - an empire predating this era by centuries. This finding is forcing historians to rethink a crucial period in the development of astronomy. It may well be that geared devices such as the Antikythera mechanism did not model the Greeks' geometric view of the cosmos after all. They inspired it.

The remains of the Antikythera mechanism were salvaged from a shipwreck in 1901 (see 'Celestial mirror from the deep') and are now held in the National Archaeological Museum in Athens. A series of ever more sophisticated radiographic studies of the gearwheels hidden inside the corroded mass culminated in proposed reconstructions of the device from a team led by astronomer Mike Edmunds of the University of Cardiff, UK, in 2006 (ref. 1), and from London-based mechanic and curator Michael Wright in 2007 (ref. 2).

The device, which dates from the second or early first century вс, was enclosed in a wooden box roughly 30 centimetres high by 20 centimetres wide, contained more than 30 bronze gearwheels and was covered with Greek inscriptions. On the front was a large circular dial with two concentric scales. One, inscribed with names of the months, was divided into the 365 days of the year; the other, divided into 360 degrees, was marked with the 12 signs of the zodiac.

Pointers moving around this dial were thought to show the date as well as the corresponding position of the Sun, Moon and probably the five planets known at the time. A revolving ball, painted half black and half silver, displayed the phase of the Moon, and letters marked on the zodiac scale acted as a kind of index, linking to inscriptions that described the appearances and disappearances of major stars at different times of the year.
On the back of the device were two spiral dials, one above the other. The top one showed a repeating 235-month calendar, popular because after 235 months or 19 years, the distribution of new Moons in the solar year is the same. The bottom spiral represented a 223-month repeating eclipse cycle. Symbols inscribed on its month divisions told the user when to expect eclipses, and gave information about the type and timing of each event.

The researchers who did the 2006 reconstruction noted that the 235- and 223-month cycles were originally derived by the Babylonians. That was to be expected: the empire's priest-astronomers, who saw astronomical events as powerful omens, had identified many such cycles over the centuries, and Greek astronomers of this period often made use of their results.

But this fact did not shake the researchers' central conclusion that the device embodied the Greeks' own geometrical models of the cosmos. These models, based on spheres or circles that described the motion of the planets in three-dimensional space, had originally been qualitative and philosophically pleasing rather than accurate. But by the time of the Antikythera mechanism's construction, scholars such as Hipparchus, who worked in Rhodes in the second century $\mathrm{BC}$, had been inspired by the Babylonians' precision to put numbers into the Greek models, and to insist 
CROSS-CULTURAL COMPUTER

The latest reconstruction of the Antikythera mechanism suggests that its front display relied on Babylonian theories describing the Sun, Moon and planets.

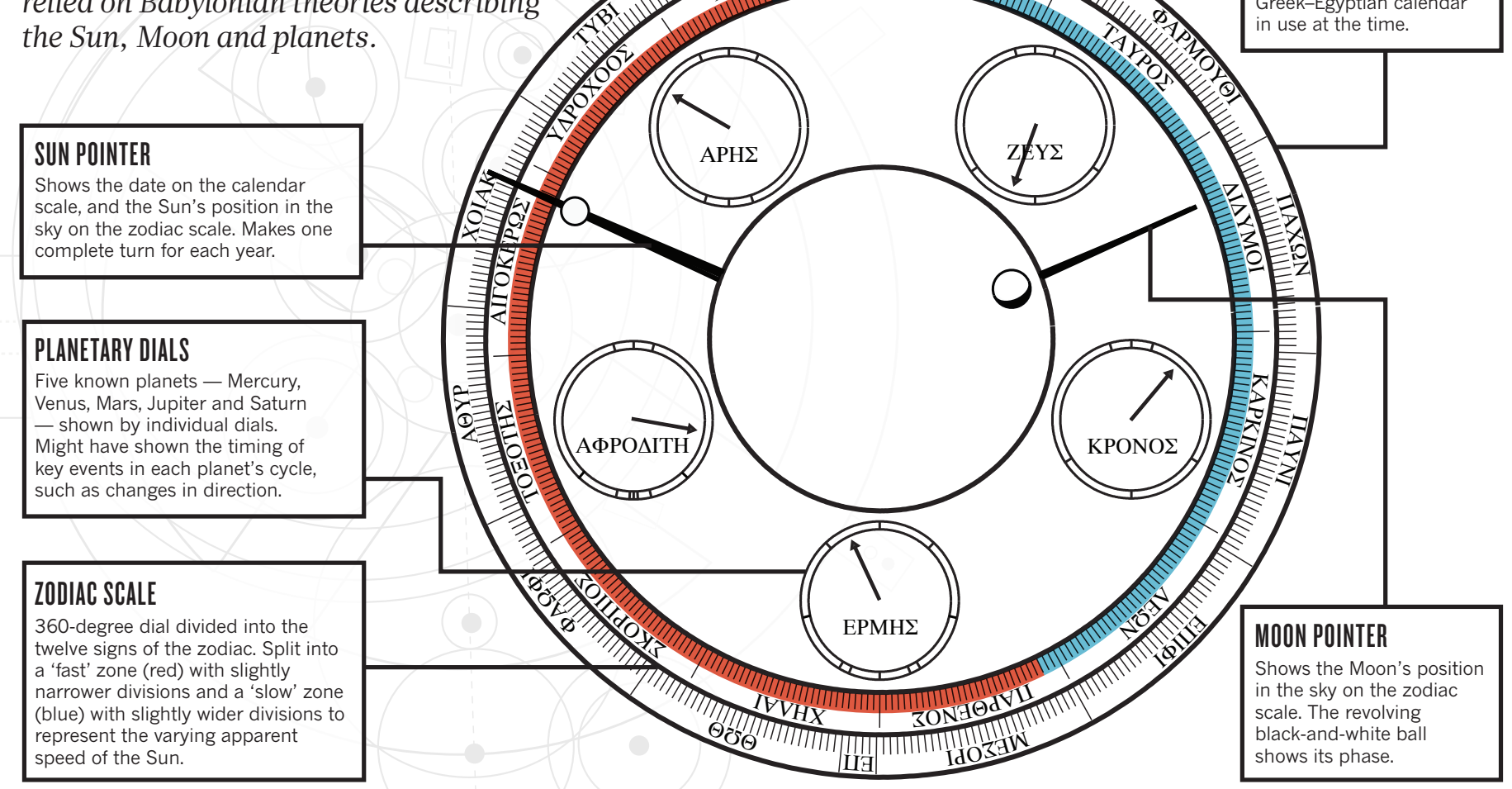

that they fit with actual observations. Modern experts were confident that the device's zodiac display - its centrepiece - reflected such state-of-the-art geometrical theories.

Supporting this idea were X-ray scans revealing that a mechanism hidden within the device's clockwork directly modelled the varying motion of the Moon. Because the Moon's orbit around Earth is elliptical rather than circular, it seems to travel faster at some points in its orbit than others. Greek philosophers believed that all heavenly orbits were perfect circles, so Hipparchus explained this variation in the Moon's motion by superimposing one circular orbit onto another that had a different centre - the 'eccentric' theory.

\section{MISSING GEARS}

The gearwork in the Antikythera mechanism seems to put this into practice perfectly, using a pin-and-slot mechanism that enabled one gearwheel to drive another around a slightly displaced axis. In their 2006 Nature paper ${ }^{1}$, Edmunds and his colleagues described it as "a mechanical realization" of Hipparchus' lunar theory. The team and other scholars assumed that the maker of the Antikythera mechanism must have used similar techniques to model the path of the Sun and probably the planets, too. The relevant gearing is missing, but the assumption is plausible: Greek astronomers accounted for the movement of planets
- which not only seem to speed up and slow down in the sky, but sometimes change direction - using a theory that was mathematically equivalent to the eccentric model. The basic idea, which would be refined and made famous by the Greek astronomer Ptolemy in the second century AD, was that each planet travelled in a small circle called an epicycle, whose centre was simultaneously moving in a larger loop around Earth.

To demonstrate how the Antikythera mechanism could have operated, Wright built a working model of it. His device incorporates small gear wheels riding on larger ones to model the epicycles of Mercury, Venus, Mars, Jupiter and Saturn, as well as the varying speed of the Sun. The Antikythera mechanism thus seemed to be a stunning demonstration of how the ancient Greeks had translated their most famous astronomical theory into physical wheels of bronze.

But now a new team has noticed a detail that could turn this view of the mechanism on its head. Historian of astronomy James Evans at the University of Puget Sound in Tacoma, Washington, and his colleagues knew that the 360 divisions on the zodiac scale should be spaced slightly farther apart than the 365 divisions on the calendar scale that encircled it. But when they used X-ray scans provided by Edmunds' team to precisely measure the division widths on the surviving part of the dial, which encompasses 88 degrees, they found that the zodiac marks are actually closer together ${ }^{3}$. The marks on the vanished parts of the scale must have compensated somehow with a wider spacing.

The researchers believe that this was done on purpose to represent the Sun's uneven progress through the sky. Instead of the device using epicyclic gearing to drive a pointer with varying speed as previously thought, Evans believes it is "extremely likely" that its maker used a pointer moving with constant speed around a circle split into two sections of equal overall size that were divided differently: a 'fast zone' in which the degree markings were closer together than normal, and a 'slow zone' in which they were farther apart. This scheme is identical to a theory of the Sun's movement used by the Babylonians, known as System A.

If correct, this interpretation suggests that the astronomy encoded in the mechanism's gearwork does not represent state-of-theart Greek theories after all. It is Babylonian through and through.

This is a tough assertion to prove. The uneven division of the zodiac scale could have been just the result of sloppy work by the machine's creator, and its similarity to the Babylonian scheme just a coincidence. Wright, who was the first to suggest that epicyclic gearing modelled the motions of the Sun and planets, says he is "very 
uncomfortable" with the idea that the device modelled the Moon's motion mechanically, yet used an abstract numerical scheme to do the same for the Sun.

But astronomy historian Alexander Jones of the Institute for the Study of the Ancient World in New York is taking the hypothesis seriously. He argues that Greek astronomers were more interested in convenience than consistency. Such an intimate mix of geometric and arithmetic approaches fits the spirit of the period, he says. "They were playing with different toolboxes at the same time."

\section{KEY EVENTS}

Evans's hypothesis forces a rethink of other parts of the mechanism, too. Previously, scholars assumed that the positions of the Sun, Moon and planets were all displayed around the same zodiac scale. But if the zodiac scale had been tweaked to accommodate the varying speed of the Sun, it would no longer be accurate for showing the positions of the other bodies.

Evans thinks that the five planets were instead displayed on individual smaller dials (see 'Cross-cultural computer'). He adds that these dials didn't necessarily have to show the planets' positions in the sky. He thinks the machine's maker would have been more interested in showing the timing of key events in each planet's cycle, such as changes of direction, or first and last appearance in the night sky. If so, the pointers on these dials could have been driven at constant speed by simple gear trains representing period relations derived by the Babylonians - no epicycles required.

Jones is more cautious about this suggestion, although he says it "makes sense in terms of how planetary motion was talked about at the time". Both he and Evans are hoping that more clues will come from inscriptions on the front cover of the mechanism. The surviving lettering is hidden inside the mechanism's battered and corroded remains, but it is being painstakingly reconstructed and translated from X-ray scans by Agamemnon Tselikas, director of the Centre for History and Palaeography in Athens, and Yanis Bitsakis, a physicist at the University of Athens. So far the two researchers have deciphered mentions of Mars, Mercury and Venus, along with several references to the 'stationary points' at which planets seem to change direction.

Evans argues that even the clearly epicyclic gearing of the Moon display may model Babylonian arithmetic, not Greek geometry. The amplitude of the variation encoded by the pin-and-slot mechanism is larger than that used by Hipparchus in his eccentric model, he points out, and is closer to the amplitude used in the lunar algorithms of the Babylonians. "Perhaps a mechanic tried to represent the variations in the Moon's speed according to the Babylonian theory using gears," he says and hit upon an epicyclic arrangement.

In other words, epicycles were not a philosophical innovation but a mechanical one. Once Greek astronomers realized how well epicyclic gearing in devices such as the Antikythera mechanism replicated the cyclic variations of celestial bodies, they could have incorporated the concept into their own geometrical models of the cosmos.

"It is a new possibility," says Jones. "I am quite attracted to it." There is little evidence for who came up with the idea of epicycles, although it is often ascribed to third-century-BC Greek geometer Apollonius of Perga. Intriguingly, gears and epicycles seem to have arisen at about the same time, with gears perhaps a little earlier. Also in the third century BC, Archimedes used simple gears to change the size of an applied force. Some two centuries later, the Roman politician and author Cicero wrote that Archimedes built a bronze astronomical device that might have been similar to the Antikythera mechanism.

"Maybe we need to rethink the connection between mechanics and astronomy," says Evans. "People think of it as purely one way, but maybe there was more of an interplay." In other words, when that Greek mechanic shaped the Antikythera mechanism's complex gear trains, he created more than a model made of bronze. He helped to forge a view of the Universe that would hold sway for nearly 2,000 years.

Jo Marchant is a London-based writer and author of Decoding the Heavens, a book about the Antikythera mechanism.

1. Freeth, T. et al. Nature 444, 587-591 (2006).

2. Wright, M. T. Interdiscipl. Sci. Rev. 32, 27-43 (2007)

3. Evans, J., Carman, C. C. \& Thorndike, A. S. J. Hist. Astron. 41, 1-39 (2010).

4. Freeth, T., Jones, A., Steele, J. M. \& Bitsakis Y. Nature 454, 614-617 (2008)

5. Højte, J. M. (ed.) Mithridates VI and the Pontic Kingdom 313-320 (Aarhus Univ. Press, 2009).

\section{CELESTIAL MIRROR FROM THE DEEP}

\section{Where did the Antikythera mechanism come from?}

\section{In 1900, when a crew of}

Mediterranean sponge divers took shelter from a storm by the tiny island of Antikythera (see inset map), they found an ancient wreck full of statues, jewellery, weapons, furniture and other treasure - including an odd-looking clockwork device: the Antikythera mechanism.

Scholars have been trying to reconstruct its story ever since. Studies of the wreck suggest that the vessel was Roman and had sailed from the eastern Mediterranean around 70-60 BC (see main map). The Romans were fighting King Mithridates IV of Pontus in Asia Minor at the time, so the sunken ship may well have been carrying spoils from that war.

The Antikythera mechanism was initially thought to have been made on Rhodes, where the ship had almost certainly stopped, and where the Greek astronomer Hipparchus had worked. But the month names on the device are most closely related to those used in colonies established by the city-state of Corinth in northwest Greece and Sicily ${ }^{4}$. And a four-year dial on the device displays the timing of the Olympics and various other games - including 'Naa', a small athletic event of interest only near Dodona in northwestern Greece.

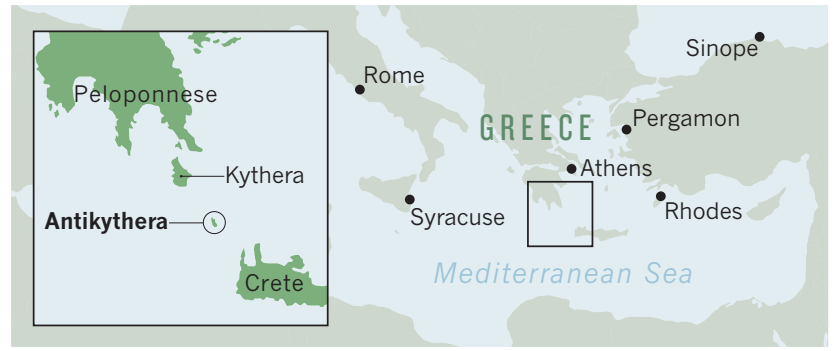

This suggests an origin (or a customer) in that vicinity. Confirmation may yet come from Magdalini Anastasiou at the University of Thessaloniki, Greece, and her colleagues, who are using the order of events listed in the device's star calendar to work out the latitude at which it was intended to be used.

Wherever it originated, the mechanism ended up in the East. Historian Attilio Mastrocinque at the University of Verona, Italy, thinks it was looted from Mithridates' capital of Sinope on the southern shore of the Black Sea ${ }^{5}$. According to the ancient Greek geographer Strabo, he notes, the Roman general Lucullus seized a mysterious "Globe of Billarus" when he conquered Sinope in 72-71 BC. This globe was never heard of again. Mastrocinque believes that this is because it sank to the bottom of the sea at Antikythera. J.M. 